\title{
CIDADES INTELIGENTES: UM ESTUDO BIBLIOMÉTRICO SOBRE A PRODUÇÃO CIENTÍFICA DA ÚLTIMA DÉCADA
}

\author{
Lauriene Teixeira Santos (UNIVERSIDADE FEDERAL DE LAVRAS) \\ lauriene.tsantos@gmail.com \\ Augusto Chaves Martins (UNIVERSIDADE FEDERAL DE LAVRAS) \\ augustoch@live.com \\ Letícia Bettoni Siqueira (UNIVERSIDADE FEDERAL DE LAVRAS) \\ leticialbs@hotmail.com \\ Izadora Ribeiro e Garcia de Oliveira (UNIVERSIDADE FEDERAL DE LAVRAS) \\ izadora_rgo@hotmail.com \\ José Augusto Oliveira (UNIVERSIDADE FEDERAL DE LAVRAS) \\ jaugusto.oliveira@outlook.com
}

\section{Resumo}

O recente conceito de cidades inteligentes surgiu a partir da necessidade de tornar a vida em sociedade mais sustentável em um ambiente que sofre constantes mudanças, que são os grandes centros urbanos, unindo assim dois fatores já emergentes no estudo acadêmico: urbanização e tecnologia. Este estudo teve como objetivo mapear o campo de estudo sobre cidades inteligentes, através de uma revisão bibliométrica. Esta foi realizada no dia 11/01/2020, na base de dados Web of Science, onde os resultados obtidos foram provenientes da seguinte busca avançada: $T I=($ smart_city $O R$ smart_cities $)$. Com o desenvolvimento do presente trabalho foi possível identificar que as publicações e citações referentes ao tema pesquisado aumentaram muito nos últimos três anos, os autores da China são os que detém maior número de artigos publicados, no entanto, são os EUA que possuem mais periódicos entre os dez que mais publicaram sobre o assunto. Além disso, foi possível identificar também a formação de seis clusters de palavras-chave acerca do tema.

Palavras-chave: Cidades inteligentes. Revisão bibliométrica. Panorama da área de estudo.

\section{Introdução}

O processo de urbanização, que tem ocorrido de forma crescente, acarretou um aumento do número de pessoas que residem em cidades, onde mais da metade da população mundial reside nos centros urbanos (PIEKAS et al., 2018). Além disso, é inegável que ao longo dos anos, houve grandes avanços na área de tecnologia, fazendo com que esta esteja presente no 
cotidiano das pessoas, mudando completamente a configuração de diversas atividades humanas.

É a combinação desses dois fatores, urbanização e tecnologia, que emergem discussões acerca de cidades inteligentes, onde busca-se a integração de pessoas e a utilização de informações, para que haja uma melhora na qualidade de vida (PIEKAS et al., 2018). De acordo com Cury e Marques (2017) o surgimento de cidades inteligentes está atrelado a busca por alternativas que busquem tornar a vida das pessoas mais sustentável, em um ambiente que sofre constantes mudanças.

Assim, através de políticas que visam a transformação do território, há a estimulação para que os cidadãos participem de forma efetiva, além de se buscar a utilização de inteligências distintas: humana, coletiva e artificial (CURY; MARQUES, 2017). A utilização da inteligência artificial acontece pela utilização das Tecnologias de Informação e Comunicação (TICs), e o termo cidade inteligente está ligado com as abordagens geográficas contemporâneas de desenvolvimento urbano (CURY; MARQUES, 2017).

Ainda que o conceito de cidade inteligente possa ser considerado relativamente recente, é possível dizer que este assunto já se encontra consolidado, mostrando-se fundamental dentre as discussões sobre desenvolvimento sustentável (FGV PROJETOS, 2019). Além disso, movimenta um mercado global de solução tecnológica, podendo chegar em US\$ 408 bilhões no ano de 2020 (FGV PROJETOS, 2019).

Nesse sentido, o objetivo do presente artigo é mapear o campo de estudo sobre cidades inteligentes, a partir de uma revisão bibliométrica. Justifica-se a realização do presente estudo, visto que há relevância nas discussões acerca das cidades inteligentes, além de que o tema ainda pode ser considerado recente. Uma revisão bibliométrica, pode então, contribuir para um melhor conhecimento sobre o que tem sido produzido academicamente sobre a temática.

A estrutura do artigo conta com outras quatro seções, além da presente introdução. A segunda seção apresenta o referencial teórico, buscando abordar de forma mais detalhada sobre cidades inteligentes. Na terceira seção, apresenta-se os procedimentos metodológicos. Posteriormente, apresenta-se os resultados da análise bibliométrica realizada. Em um último momento, são apresentadas as considerações finais do estudo e suas limitações. 


\section{Cidades inteligentes}

Devido as transformações que vêm acontecendo nas cidades, ocorreu um aumento das discussões sobre o papel destas na economia e sua influência no bem-estar social (CÂMARA et al., 2016). Para Cury e Marques (2017) um dos desafios a ser enfrentado no século XXI, encontra-se relacionado com a gestão de um ambiente sustentável, onde se preze pela qualidade de vida dos cidadãos e as particularidades de cada ecossistema. Dessa forma, os "avanços tecnológicos têm possibilitado a criação e identificação de inúmeras oportunidades para o enfrentamento dos principais problemas que afetam as cidades do presente e do futuro" (WEISS; BERNARDES; CONSONI, 2017, p. 6).

Quando se considera o futuro das cidades, não há como ignorar que a inovação tecnológica tem um papel importante a ser desempenhado, principalmente por apresentar uma demanda que envolve diferentes competências e especializações, englobando diversos tipos de profissionais na execução das mais variadas atribuições (WEISS; BERNARDES; CONSONI, 2017). Para os autores, a junção de competências profissionais e tecnologia é fator essencial para que as cidades prosperem, sendo fundamental haver uma avaliação e entendimento sobre as características e necessidades dessas cidades.

De acordo com Lemos e Araújo (2018) os projetos relacionados a cidades inteligentes, ainda se encontram em fase de especulação, onde há diversas promessas e poucas experiências concretas que possam servir de base para a implementação de demais projetos. Contudo, os autores destacam que, apesar disso, as grandes cidades do mundo, estão aderindo ao movimento, em maior ou menor escala.

Associa-se o termo cidade inteligente, a utilização eficiente de TICs visando melhorar a infraestrutura e os serviços de uma cidade, onde dispende-se uma maior atenção pela eficiência dos serviços urbanos e agilidade nas operações de forma a atenuar assimetrias, gerando benefícios para todos os cidadãos (PIEKAS et al., 2018).

Dessa forma, as cidades inteligentes possuem como objetivo central servir aos seus habitantes, destinando-se a melhorar o bem-estar da população em geral (CÂMARA et al., 2016). "O adjetivo "inteligência" é entendido como o processamento automático da informação e a análise de um grande volume de dados" (LEMOS; ARAÚJO, 2018, p. 3).

As cidades inteligentes fornecem serviços interoperáveis, que facilitam a conectividade para transformar processos de governo internamente entre as agências e departamentos e, externamente para cidadãos e empresas. Para criar cidades inteligentes, as tecnologias devem estar prontamente integradas, conectando 
diferentes sistemas em diferentes organizações. Nas cidades inteligentes, a tecnologia da informação desempenha o papel de tornar os dados da vida urbana tangíveis, por meio da criação e execução de projetos voltados para a sua captura e tratamento em tempo real (WEISS; BERNARDES; CONSONI, 2017, p. 6).

Todavia, para que o projeto de uma cidade inteligente seja implementado com sucesso, existe a necessidade de haver melhorias em diversos aspectos. Câmara et al. (2016) apontam que é preciso reduzir a pobreza, buscando que o número de pessoas com acesso à tecnologia cresça

de forma considerável. É preciso também que se eleve o nível de educação da população, buscando capacitar os cidadãos para que saibam utilizar e conservar a tecnologia disponibilizada, já que é a tecnologia que permite a inovação das cidades (CÂMARA et al., 2016).

Conforme os acadêmicos reconhecerem que além das TICs, fatores como capital humano, educação e questões ambientais também contribuem para a melhoria de uma cidade, ampliouse o conceito de cidade inteligente (BRANDÃO; JOIA, 2018). Neste ponto, é que se distingue uma cidade digital de uma cidade inteligente, uma vez que na cidade inteligente há a necessidade de realizar a inclusão do fator humano, onde não é suficiente apenas a existência de uma estrutura tecnológica (CURY; MARQUES, 2017). Para os autores, as pessoas são capazes e habilitadas a propor soluções para os problemas que enfrentam no dia a dia, se mostrando então, "o elo principal por disporem de informações únicas e particulares advindas de suas percepções sobre o ambiente em que vivem” (CURY; MARQUES, 2017, p. 104).

Assim sendo, a cidade torna-se um laboratório vivo, onde a população precisa envolver-se no desenvolvimento de testes de soluções inovadoras (CÂMARA et al., 2016). Ainda de acordo com os autores, experiências positivas podem ser replicadas ou adaptadas para outros territórios, onde os projetos podem ser desenvolvidos pelo governo, por acadêmicos e pela população.

Através de um empenho conjunto das inteligências humana, coletiva e artificial é que se constrói uma cidade inteligente, onde o aparato intelectual é valorizado a partir da capacidade de inovação de seus agentes, que criam e implementam soluções tecnológicas, desenvolvendo um espaço em que acontece uma aprendizagem constante (CURY; MARQUES, 2017).

\section{Procedimentos metodológicos}

A presente pesquisa se caracteriza como um estudo bibliométrico, onde este consiste em uma avaliação objetiva da produção científica de determinada área de estudo, através da utilização 
de métodos quantitativos (ARAÚJO, 2006). De acordo com o autor, a partir da bibliometria é possível identificar e descrever diversos padrões existentes na produção do conhecimento científico. Quando se trata do campo específico das ciências socias aplicadas, "os estudos bibliométricos se concentram em examinar a produção de artigos em um determinado campo de saber, mapear as comunidades acadêmicas e identificar as redes de pesquisadores e suas motivações" (CHUEKE; AMATUCCI, 2015, p. 2).

Dessa forma, a base de dados selecionada foi a Web of Science (WoS) - Coleção Principal, por duas razões: é uma base de dados que indexa publicações científicas de todo mundo, contendo mais de 11.000 periódicos disponíveis (FUNDAÇÃO CAPES, 2011, 2014).

Após da escolha da base de dados, passou-se para a escolha dos termos de busca. Optou-se por buscar pelo termo "Cidade Inteligente" na sua forma singular e plural, objetivando não excluir nenhum artigo pela terminologia utilizada. Além disso, optou-se pela busca de trabalhos que contivessem alguns dos termos (singular ou plural) em seu título. Com isso, acredita-se que a busca realizada foi pertinente, no sentido de não abranger trabalhos que apresentassem o termo no corpo do texto, mas que não o tratassem como o foco principal da discussão gerada no artigo.

A busca foi realizada no dia 11/01/2020, e os resultados encontrados foram provenientes da seguinte busca avançada: $T I=($ smart_city $O R$ smart_cities $)$. Realizou-se também um recorte temporal, buscando apenas artigos dos últimos dez anos (2009-2019), objetivando realizar uma pesquisa bibliométrica que contemplasse o estado da arte do tema "Cidade Inteligente", apresentando dados dos artigos mais recentes. Estes poderiam estar em qualquer idioma e em qualquer área de conhecimento.

Por fim, optou-se por trabalhar apenas com artigos científicos, com o intuito de encontrar trabalhos que possuíssem uma discussão mais consolidada sobre o tema. Na Tabela 1 , apresenta-se a sequência de escolhas realizadas para buscar os artigos na Web of Science.

Tabela 1 - Síntese dos parâmetros de busca

\begin{tabular}{|c|c|}
\hline Tipo de pesquisa & Revisão bibliométrica \\
\hline Base de dados & Web of Science \\
\hline Termo de busca & TI=(smart_city OR smart_cities) \\
\hline Devem aparecer & Somente no título \\
\hline Recorte temporal & 2009 até 2019 \\
\hline Idioma & Sem definição \\
\hline Área de conhecimento & Sem definição \\
\hline Tipo de documento & Somente artigos \\
\hline
\end{tabular}

Fonte: Elaborado pelos autores (2020) 
Após a execução da pesquisa no WoS, chegou-se ao número de 1648 artigos. Realizou-se a exclusão de 5 artigos, uma vez que estes estavam duplicados. Dessa forma, os artigos foram analisados com o auxílio dos softwares EndNote Web, Microsoft Excel e VOSviewer, buscando a organização dos documentos e a construção de gráficos e mapas bibliométricos.

A seguir, apresenta-se as análises realizadas a partir dos 1643 artigos selecionados, sendo que estas foram direcionadas para a quantidade de publicações por ano; áreas de publicação; países que mais publicaram; idioma dos artigos; periódicos mais relevantes conforme o número de publicações; periódicos mais relevantes conforme o número de citações; autores mais produtivos; e, artigos mais relevantes quanto ao número de citações.

\section{Resultados e discussão}

Nota-se que os dois primeiros artigos relacionados ao tema, só foram publicados no ano de 2010, conforme a Gráfico 1. Este fato torna-se condizente, pois conforme abordado na introdução, este tema é relativamente recente. No entanto, o pouco tempo de estudo deste assunto não o torna menos relevante, uma vez que a partir de 2010 o número de publicações só cresceu, chegando ao número de 555 artigos publicados somente em 2019.

Gráfico 1 - Evolução temporal das publicações

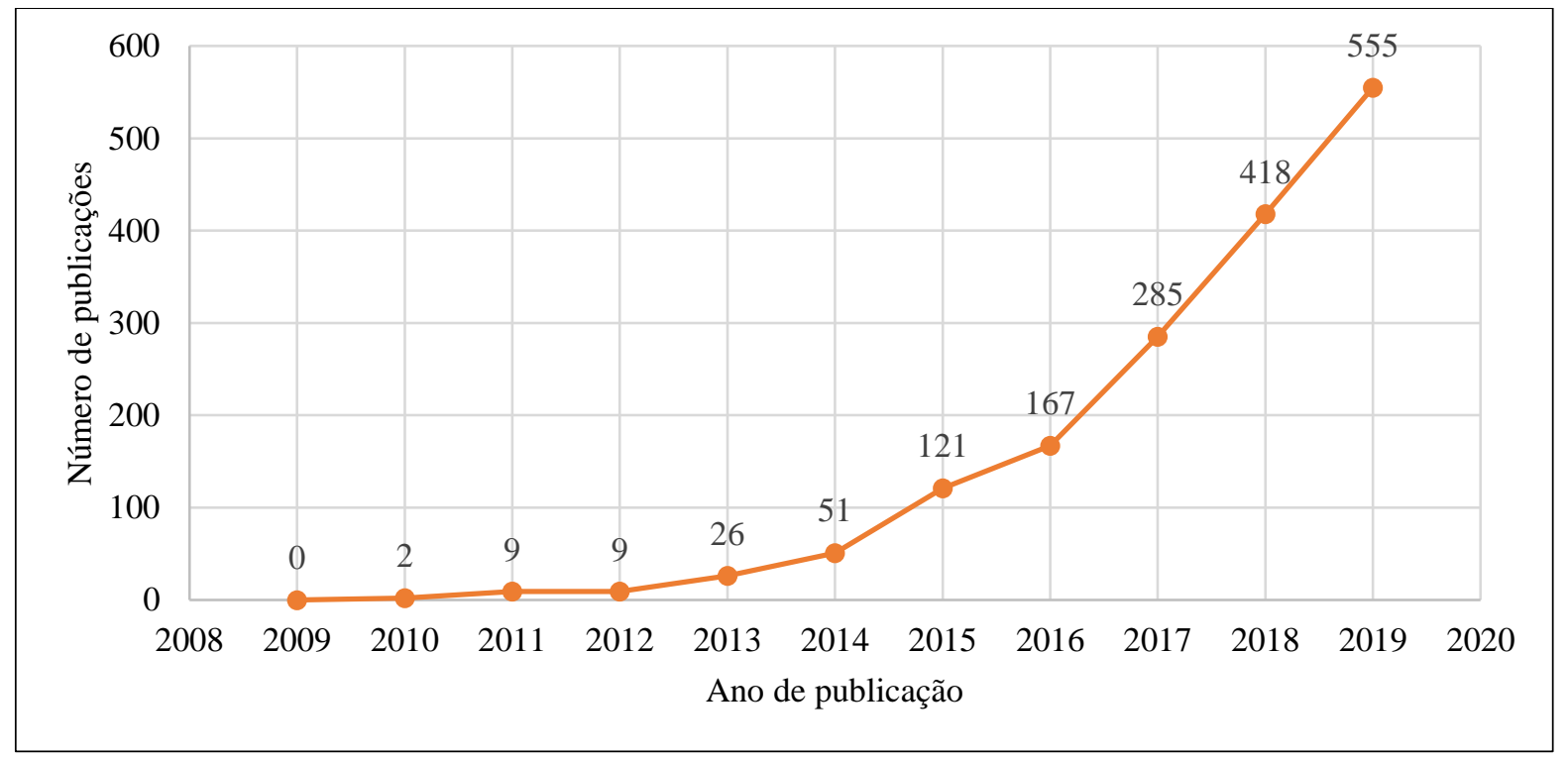

Fonte: Elaborado pelos autores (2020)

Destaca-se ainda que nos últimos três anos houve um grande aumento pelo interesse do campo estudado, uma vez que foram publicados 1.258 artigos entre 2017 e 2019, o que 
representa $76,57 \%$ do total de publicações. Além disso, ainda neste período, os artigos da frente de pesquisa receberam 18.183 citações, $84,5 \%$ do total, como mostra a Gráfico 2.

Gráfico 2 - Evolução temporal das citações

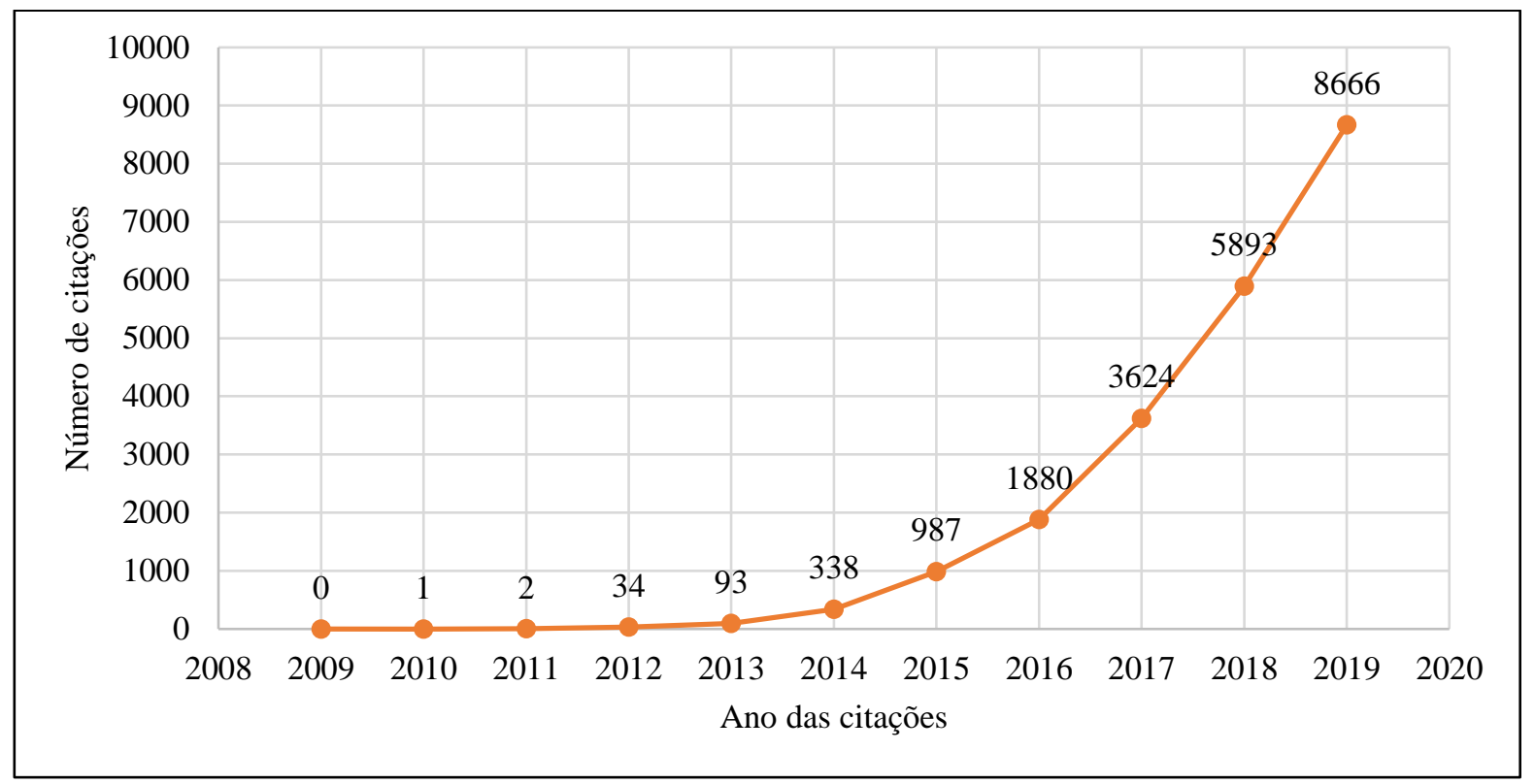

Fonte: Elaborado pelos autores (2020)

Ao analisar os Gráficos 1 e 2, percebe-se a semelhança entre a evolução de publicações e citações, onde o número de publicações impacta diretamente o número de citações. A seguir, na Tabela 2, apresentam-se os 10 periódicos que mais publicaram sobre o tema da pesquisa.

Tabela 2 - Periódicos que mais publicaram sobre os termos de busca na base WoS

\begin{tabular}{ccccc}
\hline$\#$ & Periódico & JRC (2018) & País & $\mathbf{N}^{\mathbf{0}}$ de publicações \\
\hline 1 & Sensors & 3.031 & Switzerland & 82 \\
2 & IEEE Acess & 4.098 & USA & 81 \\
3 & IEEE Communications Magazine & 10.356 & USA & 49 \\
4 & $\begin{array}{c}\text { Future Generation Computer Systems } \\
\text { the International Journal of Escience }\end{array}$ & 5.768 & Netherlands & 48 \\
5 & Sustainability & 2.592 & Switzerland & 40 \\
6 & IEEE Internet of Things Journal & 9.515 & USA & 37 \\
7 & Technological Forecasting and & 3.815 & USA & 35 \\
8 & Social Change & & \\
9 & Cities & 3.853 & England & 31 \\
10 & Journal of Urban Technology & 2.25 & Netherlands & 28 \\
\hline
\end{tabular}

Fonte: Elaborado pelos autores (2020)

Dentre eles, destaca-se o Sensors da Suíça, com o maior número de publicações (82). Observa-se que dos 10 periódicos que mais publicaram sobre o assunto, 4 são sediados nos 
EUA, 2 na Suíça, 2 nos Países Baixos e 2 na Inglaterra. Ainda de acordo com a Tabela 2, é possível perceber que o periódico com maior JCR (IEEE Communications Magazine), encontra-se na terceira colocação dentre os periódicos que mais publicaram sobre essa temática.

A Tabela 3 apresenta os 10 artigos mais citados (citações indicadas na base $W o S$ ) da frente de pesquisa. Juntos, estes artigos receberam 5.365 citações de um total de 21.518, ou seja, $24,93 \%$.

Tabela 3 - Artigos mais citados relacionados aos termos de busca na base WoS

\begin{tabular}{|c|c|c|c|c|c|c|}
\hline \# & Título & Autores & Periódicos & $\begin{array}{c}\text { Ano de } \\
\text { publicação }\end{array}$ & $\begin{array}{l}\mathrm{N}^{\circ} \text { total } \\
\text { de } \\
\text { citações }\end{array}$ & $\begin{array}{c}\text { Média de } \\
\text { citações/ano }\end{array}$ \\
\hline 1 & $\begin{array}{l}\text { Internet of } \\
\text { Things for } \\
\text { Smart Cities }\end{array}$ & $\begin{array}{c}\text { Zanella, A.; Bui, N.; } \\
\text { Castellani, A.; } \\
\text { Vangelista, L.; Zorzi, } \\
\text { M. }\end{array}$ & $\begin{array}{l}\text { IEEE INTERNET OF } \\
\text { THINGS JOURNAL }\end{array}$ & 2014 & 1541 & 220,14 \\
\hline 2 & $\begin{array}{l}\text { Smart Cities in } \\
\text { Europe }\end{array}$ & $\begin{array}{r}\text { Caragliu, A.; Del } \\
\text { Bo, C.; Nijkamp, P. }\end{array}$ & $\begin{array}{l}\text { JOURNAL OF URBAN } \\
\text { TECHNOLOGY }\end{array}$ & 2011 & 770 & 77 \\
\hline 3 & $\begin{array}{l}\text { Current trends } \\
\text { in Smart City } \\
\text { initiatives: } \\
\text { Some stylised } \\
\text { facts }\end{array}$ & $\begin{array}{c}\text { Neirotti, P.; De } \\
\text { Marco, A.; Cagliano, } \\
\text { A. C.; Mangano, G.; } \\
\text { Scorrano, F. }\end{array}$ & CITIES & 2014 & 526 & 75,14 \\
\hline 4 & $\begin{array}{l}\text { Smart cities of } \\
\text { the future }\end{array}$ & $\begin{array}{l}\text { Batty, M.; Axhausen, } \\
\text { K. W.; Giannotti, } \\
\text { F.; Pozdnoukhov, } \\
\text { A.; Bazzani, A.; } \\
\text { Wachowicz, } \\
\text { M.; Ouzounis, } \\
\text { G.; Portugali, Y. }\end{array}$ & $\begin{array}{c}\text { EUROPEAN } \\
\text { PHYSICAL JOURNAL- } \\
\text { SPECIAL TOPICS }\end{array}$ & 2012 & 490 & 54,44 \\
\hline 5 & $\begin{array}{l}\text { Smart Cities: } \\
\text { Definitions, } \\
\text { Dimensions, } \\
\text { Performance, } \\
\text { and Initiatives }\end{array}$ & $\begin{array}{l}\text { Albino, V.; Berardi, } \\
\text { U.; Dangelico, R. M. }\end{array}$ & $\begin{array}{l}\text { JOURNAL OF URBAN } \\
\text { TECHNOLOGY }\end{array}$ & 2015 & 456 & 76 \\
\hline 6 & $\begin{array}{l}\text { An Information } \\
\text { Framework for } \\
\text { Creating a } \\
\text { Smart City } \\
\text { Through } \\
\text { Internet of } \\
\text { Things }\end{array}$ & $\begin{array}{c}\text { Jin, J. ; Gubbi, } \\
\text { J.; Marusic, } \\
\text { S.; Palaniswami, M. }\end{array}$ & $\begin{array}{l}\text { IEEE INTERNET OF } \\
\text { THINGS JOURNAL }\end{array}$ & 2014 & 433 & 61,86 \\
\hline
\end{tabular}




\begin{tabular}{|c|c|c|c|c|c|c|}
\hline 7 & $\begin{array}{l}\text { Sensing as a } \\
\text { service model } \\
\text { for smart cities } \\
\text { supported by } \\
\text { Internet of } \\
\text { Things }\end{array}$ & $\begin{array}{c}\text { Perera, } \\
\text { C.; Zaslavsky, } \\
\text { A.; Christen, P.; } \\
\text { Georgakopoulos, D. }\end{array}$ & $\begin{array}{c}\text { TRANSACTIONS ON } \\
\text { EMERGING } \\
\text { TELECOMMUNICATI } \\
\text { ONS } \\
\text { TECHNOLOGIES }\end{array}$ & 2014 & 355 & 50,71 \\
\hline 8 & $\begin{array}{c}\text { Smartmentality: } \\
\text { The Smart City } \\
\text { as Disciplinary } \\
\text { Strategy }\end{array}$ & Vanolo, A. & URBAN STUDIES & 2014 & 278 & 39,71 \\
\hline 9 & $\begin{array}{l}\text { Foundations for } \\
\text { Smarter Cities }\end{array}$ & $\begin{array}{c}\text { Harrison, } \\
\text { C.; Eckman, } \\
\text { B.; Hamilton, } \\
\text { R.; Hartswick, } \\
\text { P.; Kalagnanam, } \\
\text { J.; Paraszczak, } \\
\text { J.; Williams, P. }\end{array}$ & $\begin{array}{l}\text { IBM JOURNAL OF } \\
\text { RESEARCH AND } \\
\text { DEVELOPMENT }\end{array}$ & 2010 & 275 & 25 \\
\hline 10 & $\begin{array}{l}\text { Long-range } \\
\text { communications } \\
\text { in unlicensed } \\
\text { bands: the } \\
\text { rising stars in } \\
\text { the iot and } \\
\text { smart city } \\
\text { scenarios }\end{array}$ & $\begin{array}{c}\text { Centenaro, } \\
\text { M.; Vangelista, } \\
\text { L.; Zanella, } \\
\text { A.; Zorzi, M. }\end{array}$ & $\begin{array}{c}\text { IEEE WIRELESS } \\
\text { COMMUNICATIONS }\end{array}$ & 2016 & 241 & 48,2 \\
\hline
\end{tabular}

Fonte: Elaborado pelos autores (2020)

Vale destacar também que, 5 dos 10 artigos mais citados estão publicados em 3 dos periódicos que mais publicaram conforme a Tabela 2, são eles: Internet of Things for Smart Cities e An Information Framework for Creating a Smart City Through Internet of Things publicados no periódico IEEE Internet of Things Journal, Smart Cities in Europe e Smart Cities: Definitions, Dimensions, Performance and Initiatives no periódico Journal of Urban Technology, e Current trends in Smart City initiatives: Some stylised facts no periódico Cities.

A Tabela 4 apresenta os 16 autores que mais publicaram sobre o tema pesquisado. Observa-se que em primeiro lugar encontra-se Zhang, Y. com um total de dezesseis publicações, seguido por Munoz, L. com treze. É válido destacar também que um total de nove autores possuem sete publicações cada.

Tabela 4 - Autores que mais publicaram sobre os termos de busca na base WoS

\begin{tabular}{ccc}
\hline$\#$ & Autores & Número de artigos \\
\hline 1 & Zhang, Y. & 16 \\
2 & Munoz, L. & 13 \\
3 & Kitchin, R. & 10
\end{tabular}




\begin{tabular}{ccc}
4 & Song, H. B. & 9 \\
5 & Wu, J. & 9 \\
6 & Kantarci, B. & 8 \\
7 & Sanchez, L. & 8 \\
8 & Angelidou, M. & 7 \\
9 & Deakin, M. & 7 \\
10 & Kumar, N. & 7 \\
11 & Park, J. H. & 7 \\
12 & Paul, A. & 7 \\
13 & Santana Jr. & 7 \\
14 & Sotres, P. & 7 \\
15 & Wang, T. & 7 \\
16 & Yigitcanlar, T. & 7 \\
\hline
\end{tabular}

Fonte: Elaborado pelos autores (2020)

Ainda com relação aos autores que mais possuem quantidade de artigos publicados sobre o tema estudado, cabe fazer um paralelo com os autores dos artigos mais citados, visto que nenhum dos autores com maior quantidade de artigos possuem algum destes dentre os mais citados. Isso pode demonstrar que nem sempre a quantidade de artigos publicados por um autor, sobre determinado assunto, implica em um grande impacto na área de estudo.

Já a Tabela 5 mostra as quinze áreas da ciência que mais publicaram acerca do tema pesquisado. Em primeiro lugar encontra-se a Engenharia Elétrica e Eletrônica com um total de 407 artigos publicados, seguida pela área de Telecomunicações com 367 e Sistemas de Informação da Ciência da Computação com 354. Em penúltimo lugar encontra-se a área de Ciências Ambientais com 77 publicações e por último a área de Gerenciamento com apenas 72 artigos publicados.

Tabela 5 - Áreas que mais publicaram sobre os termos de busca na base WoS

\begin{tabular}{ccc}
\hline$\#$ & Área de publicação & Número de artigos \\
\hline 1 & Engineering Eletrical Eletronic & 407 \\
2 & Telecommunications & 367 \\
3 & Computer Science Information Systems & 354 \\
4 & Computer Science Theory Methods & 140 \\
5 & Urban Studies & 109 \\
6 & Instruments Instrumentation & 100 \\
7 & Green Sustainable Science Technology & 96 \\
8 & Environmental Studies & 95 \\
9 & Computer Science Software Engineering & 93 \\
10 & Computer Science Hardware Architectue & 84 \\
11 & Chemistry Analytical & 82 \\
12 & Computer Science Interdisciplinary Applications & 79
\end{tabular}


$14 \quad$ Environmental Sciences $\quad 77$

Fonte: Elaborado pelos autores (2020)

A Figura 1 apresenta a distribuição geográfica dos artigos publicados com os termos pesquisados. Dentre elas, os principais países em volume de publicação são a China, EUA, Itália, Espanha e Inglaterra. Observando estes países, pode-se verificar que, de acordo com a Web of Science, a China possui 290 publicações, os EUA possuem 235, Itália 189, Espanha 183 e Inglaterra 153 publicações.

Figura 1 - Distribuição geográfica dos autores que publicaram sobre o tema na base WoS

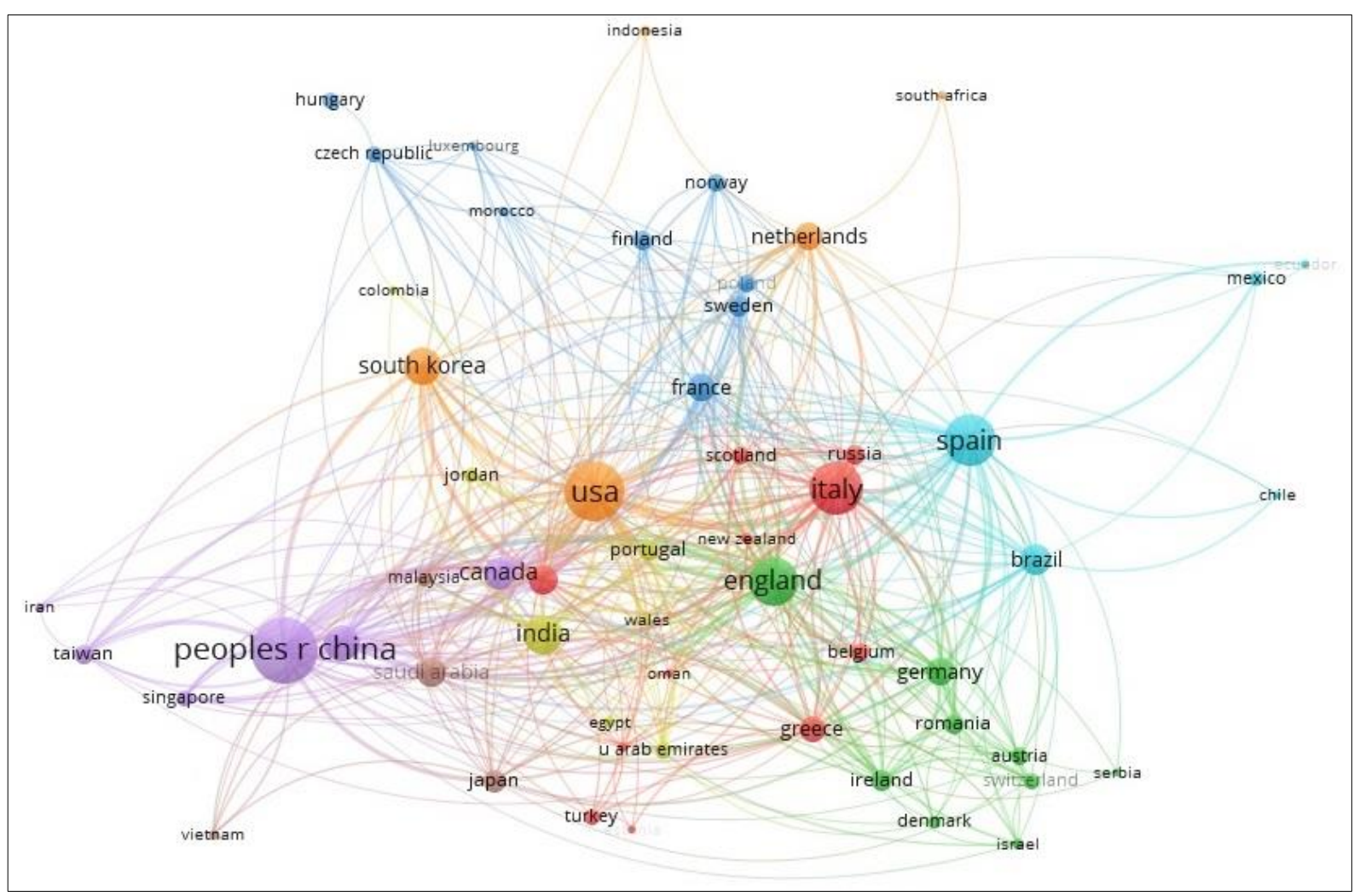

Fonte: Elaborados pelos autores (2020)

O Brasil apresentou 65 publicações relacionadas ao tema pesquisado, ocupando dessa maneira, a $10^{\mathrm{a}}$ colocação nos países que mais possuem artigos publicados acerca do assunto.

Finalmente, buscou-se construir uma rede apresentando as palavras-chave mais recorrentes nos artigos selecionados para a revisão bibliométrica (FIGURA 2). Foram contabilizados os registros com ocorrência mínima de dez casos, identificando 115 palavras no total, sendo: 41 itens no cluster vermelho, 30 no cluster alaranjado, 21 no amarelo, 18 verde, 4 no roxo e apenas 1 item no cluster azul. 
Figura 2 - Palavras-chave com maior ocorrência relacionadas aos termos de busca na base WoS

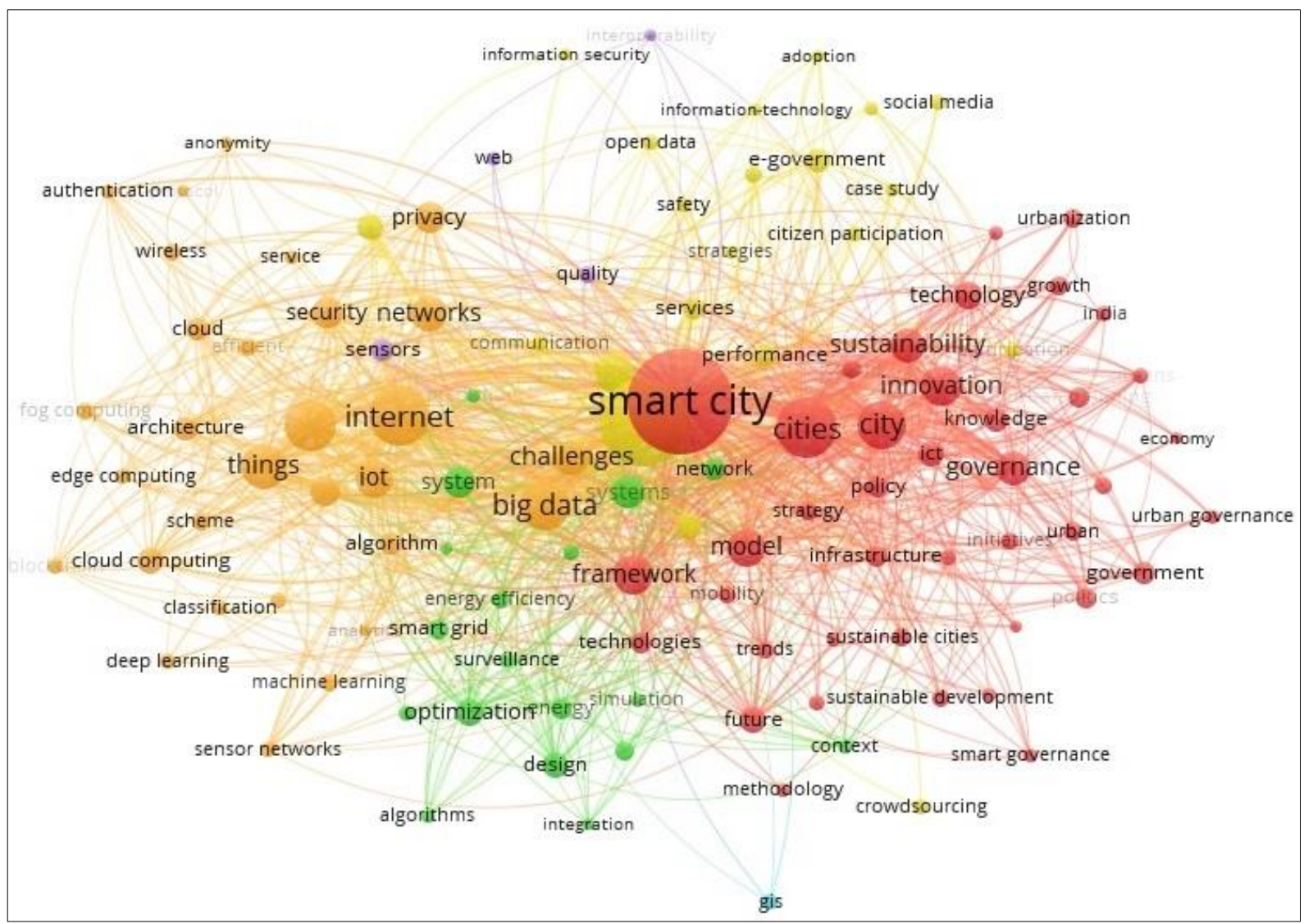

Fonte: Elaborado pelos autores (2020)

As palavras que mais apresentaram ocorrências foram: smart city (593); smart cities (422); internet (202); cities (198); internet of things (145); big data (140); city (129); things (112); innovation (90); management (86). Por fim, é possível dizer que os clusters giram em torno das palavras-chave smart city, internet, smart cities, optimization, sensors e gis, clusters vermelho, alaranjado, amarelo, verde, roxo e azul respectivamente.

\section{Considerações finais}

O presente artigo teve como objetivo mapear o campo de estudo sobre cidades inteligentes, a partir de uma revisão bibliométrica. Quanto aos estudos da frente de pesquisa, observa-se que nos últimos três anos foram publicados 1.258 artigos (76,57\% do total), reafirmando assim, o crescente interesse do meio acadêmico pelo tema. O artigo de Zanella, A. et al. (2014) é o mais citado com 1.541 citações, em seguida estão os artigos de Caragliu, A.; Del Bo, C.; Nijkamp, P. (2011) e Neirotti, P. et al. (2014) com 770 e 526 citações respectivamente.

Se tratando da distribuição geográfica dos autores que publicaram sobre o tema pesquisado, autores da China possuem 290 publicações, dos EUA possuem 235 e da Itália 189 publicações. Além disso, dos dez periódicos que mais publicam sobre o tema, quatro são 
sediados nos EUA, dois na Suíça, dois nos Países Baixos e dois na Inglaterra, sendo que os três que mais publicam são: Sensors (Suíça) com 82 artigos publicados, IEEE Acess (EUA) com 81 artigos publicados e IEEE Communications Magazine (EUA) com 49 artigos publicados.

Quanto aos autores que mais publicaram sobre o tema pesquisado, os três primeiros são Zhang, Y., Munoz, L. e Kitchin, R. com 16, 13 e 10 publicações respectivamente. Já as áreas que mais publicaram acerca do assunto, encontram-se a Engenharia Elétrica e Eletrônica, Telecomunicações e Sistemas de Informação da Ciência da Computação com 407, 367 e 354 artigos publicados respectivamente.

Por fim identificaram-se a formação de seis clusters de palavras-chave, os quais giraram em torno das palavras smart city (41 itens), internet (30 itens), smart cities (21 itens), optimization (18 itens), sensors (4 itens) e gis (apenas 1 item). Sendo assim, o cluster de palavras-chave com maior abrangência sobre o tema pesquisado é o que orbita as palavras smart city.

Ainda que a Web of Science seja uma base de dados que indexa um alto de número de periódicos, acredita-se que a utilização de uma única base de dados se apresenta como fator limitante do estudo, uma vez que a base escolhida pode não abrigar todos os artigos relacionados ao tema "Cidade Inteligente". Assim, com base nesta limitação, sugere-se para estudos futuros a utilização de diversas bases de dados, replicando os procedimentos de busca aqui realizados, buscando abranger o maior número possível de artigos sobre esta temática.

\section{REFERÊNCIAS BIBLIOGRÁFICAS}

ARAÚJO, C. A. Bibliometria: evolução histórica e questões atuais. Em questão, v. 12, n. 1, p. 11-32, 2006.

BRANDÃO, M.; JOIA, L. A. A influência do contexto na implantação de um projeto de cidade inteligente: o caso Cidade Inteligente Búzios. Revista de Administração Pública, v. 52, n. 6, p. 1125-1154, 2018.

CÂMARA, S. F.; CARVALHO, H. J. B.; SILVA, F. A. A.; SOUZA, L. L. F.; SOUZA, E. M. Cidades inteligentes no nordeste brasileiro: análise das dimensões de trajetória e a contribuição da população. Cadernos Gestão Pública e Cidadania, v. 21, n. 69, 2016.

CHUEKE, G. V.; AMATUCCI, M. O que é bibliometria? Uma introdução ao Fórum. Internext, v. 10, n. 2, p. 1 5,2015

CURY, M. J. F.; MARQUES, J. A. L. F. A cidade inteligente: uma reterritorialização. Redes - Santa Cruz do Sul, v. 22, n. 1, p. 102-117, 2017.

FGV PROJETOS, 2019. O que é uma cidade inteligente? Disponível em: https://fgvprojetos.fgv.br/noticias/oque-e-uma-cidade-inteligente. Acesso em: 08 Jan 2020. 
FUNDAÇÃO CAPES. 2014. Web of Science lança nova versão de base de dados. Disponível em: https://www.capes.gov.br/36-noticias/4484-web-of-science-lanca-nova-versao-de-base-de-dados. Acesso em: 08 Jan 2020.

LEMOS, A.; ARAÚJO, N. V. Cidadão Sensor e Cidade Inteligente: Análise dos Aplicativos Móveis da Bahia. Revista Famecos, v. 25, n. 3, p. 1-19, 2018.

PIEKAS, A. A. S.; BERNARDY, R. J.; SEHNEM, S.; FABRIS, J. Aspectos legais e percepções sobre as estratégias para cidades inteligentes e criativas: estudo da cidade de Chapecó (SC). Revista Brasileira de Gestão Urbana, v. 10, n. supl., p. 197-211, 2018.

WEISS, M. C.; BERNARDES, R. C.; CONSONI, F. L. Cidades inteligentes: casos e perspectivas para as cidades brasileiras. Revista Tecnológica da Fatec Americana, v. 5, n. 1, p. 1-13, 2017. 